SHORT REPORT

\title{
Pathological substrate for regional distribution of increased atrophy rates in progressive supranuclear palsy
}

\author{
D C Paviour, J M Schott, J M Stevens, T Revesz, J L Holton, M N Rossor, A J Lees, N C Fox
}

J Neurol Neurosurg Psychiatry 2004;75:1772-1775. doi: 10.1136/jnnp.2003.033472

\begin{abstract}
Background: Most magnetic resonance imaging (MRI) studies of progressive supranuclear palsy (PSP) are crosssectional and lack post mortem confirmation of the diagnosis. MRI features described previously in PSP correspond to regions of pathological involvement demonstrated in separate studies, but serial MRI with pathological follow up has not been undertaken.

Objective: To investigate whether regions of increased atrophy rates demonstrated in PSP during life using fluid registered serial MRI correspond with pathological findings in confirmed PSP.

Methods: A 59 year old male presented with a six month history of balance problems and dysarthria. He had a symmetrical, levodopa unresponsive akinetic-rigid syndrome with a vertical supranuclear gaze palsy. A clinical diagnosis of probable PSP was made. His disease progressed relentlessly and he died five years after onset. Two serial MRI scans undertaken during life were reviewed and fluid (non-linear) registration of the images carried out. Post mortem histopathological analysis of the brain was undertaken to definitively confirm the diagnosis and compare regional pathology with the serial imaging.

Results: Fluid registration demonstrated greatest rates of atrophy in the brainstem and frontal cortex, in keeping with the distribution of pathology seen at autopsy.

Conclusion: Fluid registration of serial MRI allows the topography and rates of regional atrophy in PSP to be delineated in life. Atrophy patterns correlated well with regional pathological load. These observations suggest that serial MRI with registration may help differentiate PSP from clinically similar conditions and supports its use as a surrogate marker of disease progression.
\end{abstract}

$\mathrm{P}$ rogressive supranuclear palsy (PSP) ${ }^{1}$ is a neurodegenerative disorder with a prevalence in the United Kingdom of 5 per $100000 .^{2}$ A definitive diagnosis requires pathological confirmation of a high density of tau positive neurofibrillary tangles (NFTs) and neuropil threads (NTs) with a distinctive predominantly subcortical distribution. $^{3}$ In its fully established classic form it presents an arresting image ${ }^{4}$ with early falls, symmetrical bradykinesia and rigidity, retrocollis, frontalis overactivity, and a vertical supranuclear gaze palsy. However, early features may be subtle and distinguishing PSP from other neurodegenerative diseases may be difficult, particularly if presentation is with parkinsonism. The sensitivity and specificity of clinical diagnostic criteria are around $80 \%$.

Over the past decade there has been considerable interest in the use of magnetic resonance imaging (MRI) as an aid to diagnosis. ${ }^{6-8}$ Cross-sectional imaging can only illustrate the extent rather than the rate of atrophy at a given point in time and subtle early changes can be masked by the biological variability in normal individuals. Many MRI studies lack the validity of pathological confirmation and to interpret the MRI findings, we need to understand the pathological correlates of the regional change. Longitudinal MRI studies allow the progression of atrophy to be demonstrated in vivo and reduce problems of interindividual variability by using subjects as their own controls. To localise change in vivo we need serial volumetric MRI. The brain may lose up to $5 \%$ of its total volume in a year ${ }^{9}$ and large volume changes result in structural readjustments. An unbiased assessment of these serial images with a high level of precision may be achieved with fluid registration. This involves matching serial brain imaging using a viscous compressible fluid model, which allows a voxel by voxel assignment of rate of atrophy. This has been validated as a useful tool for assessing regions of atrophy at different stages of Alzheimer's disease (AD)..$^{10}$

Serial volumetric imaging with pathological follow up in PSP has not been reported previously. We demonstrate the pattern of increased rates of regional atrophy in vivo in a case of PSP, using fluid registration of serial MR images, with correlation of the changes with the pathological findings at post mortem.

\section{METHODS}

A 59 year old male presented with a three month history of non-specific symptoms including fear that he might be developing dementia. His son commented that his driving skills had deteriorated. Past history included hypertension controlled with bendrofluazide and enalapril, and he drank 60 units of alcohol a week. Examination by a neurologist revealed no focal neurological signs. Over the next six months his balance deteriorated and there was a change in his speech.

He was seen at the cognitive disorders clinic at the National Hospital for Neurology and Neurosurgery. By this time he had had several falls and he felt "clumsier". His speech was slurred and he had difficulty in adjusting focus from near to distant objects. He was admitted to hospital where he was noted to have absent convergence, supranuclear vertical gaze palsy, and slow vertical saccades. Smooth pursuit was impaired and optokinetic nystagmus was sluggish with eye deviation in the direction of the slow phase. He had a cautious hesitant gait, postural instability, and bradykinesia but minimal limb rigidity. There was no improvement an hour after administration of co-beneldopa (Madopar) $250 \mathrm{mg}$. Autonomic function testing was normal. Neuropsychological testing showed that he had significant cognitive impairment particularly in the frontal domains. A diagnosis of PSP was made.

Abbreviations: $A D$, Alzheimer's disease; $G P$, globus pallidus; $L C$, locus ceruleus; NFT, neurofibrillary tangle; NT, neuropil thread; PSP, progressive supranuclear palsy; STN, subthalamic nucleus 
His condition progressed relentlessly and he developed axial rigidity, frequent falls, cervical dystonia, a worsening dysarthria, and dysphagia. He died five years after the onset of the disease, 19 months after his second scan.

\section{MR imaging}

Serial Tl-weighted volumetric MRI scans were acquired, 18 months apart, on the same 1.5 GE Signa Unit (General Electric, Milwaukee, WI) using a spoiled gradient echo technique $(256 \times 128 \times 128$ matrix, FOV $24 \times 24 \times 19.2 \mathrm{~cm}$, TR/ $\mathrm{TE} / \mathrm{NEX} / \mathrm{FLIP}=35 / 5 / 1 / 35^{\circ}$ ). This yielded 124 contiguous $1.5 \mathrm{~mm}$ thick slices. The images were processed using the MIDAS software tool. ${ }^{11}$ A semiautomated technique using intensity thresholding and a series of erosions and dilatations was applied to delineate brain tissue. Using the rigidly aligned repeat scan as a starting point, ${ }^{9}$ the fluid registration model generated a voxel-level deformation field. This field gives a measure of volume change at the voxel level from which voxel compression maps are created, using a colour overlay, to signify this volume change. ${ }^{10}$

\section{Neuropathology}

The brain (1529 g) was bisected and the right half flash frozen.

Histological examination of the fixed tissue used routine stains and immunohistochemistry for tau (AT8 1:600, Autogen Bioclear, UK), ubiquitin (1:300, Dako, UK), and $\alpha$-synuclein (1:2000, Autogen Bioclear, UK). Frozen sections $(7 \mu \mathrm{m})$ from the right anterior and posterior frontal lobes were cut and stained using tau immunohistochemistry.

\section{RESULTS}

\section{Imaging}

Cross-sectional imaging during life was reported as showing global atrophy. The fluid registered images demonstrated regional volume loss and picked out particularly high rates of atrophy in the midbrain and cerebral peduncles (fig 1) as well as the precentral gyrus (fig 2).

\section{Neuropathology}

Coronal slices from the formalin fixed left half brain, showed moderate dilatation of the lateral ventricle with atrophy of the globus pallidus (GP) and subthalamic nucleus (STN), which was discoloured. The substantia nigra (SN) and locus ceruleus (LC) were pale. There was severe neuronal loss with free pigment and gliosis in the SN. Moderate cell loss and gliosis was evident in the STN and LC.

Immunohistochemical staining for tau demonstrated NFTs and NTs in the neocortex, striatum, GP, STN, SN, LC, pontine base, and dentate nucleus. In addition, there were tau

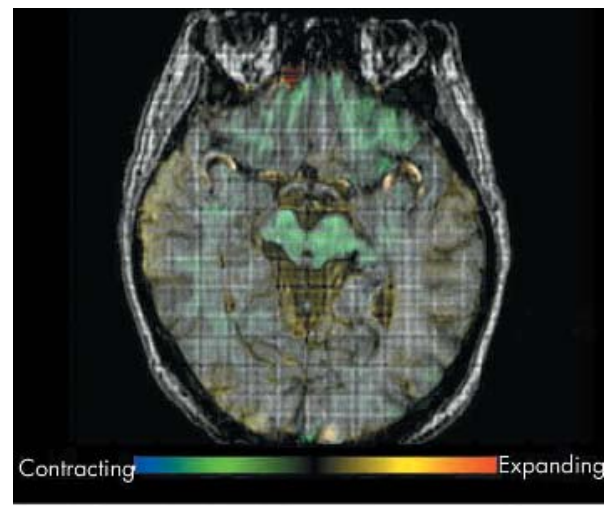

Figure 1 Axial magnetic resonance imaging scan with voxelcompression mapping overlay to demonstrate greatest rates of volume loss in the midbrain.

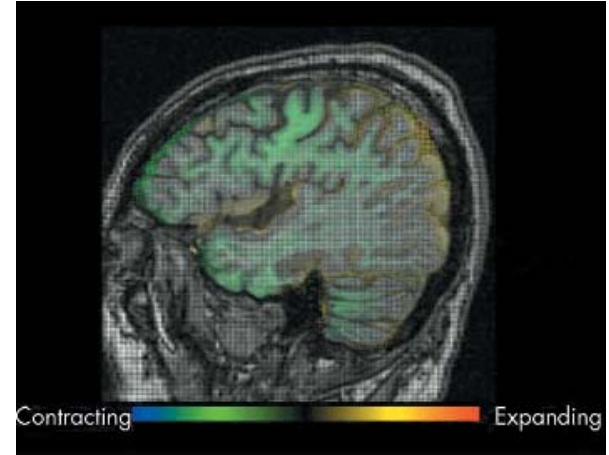

Figure 2 Sagittal magnetic resonance imaging scan with voxelcompression mapping overlay of the right hemisphere, showing volume loss affecting the frontal lobes, particularly the precentral gyrus. Concomitant expansion is seen in the cerebrospinal fluid spaces.

immunoreactive oligodendroglial inclusions particularly evident in the striatum, GP, and cerebellar white matter. Tufted astrocytes were most easily found in the frontal cortex and putamen (fig 3 A-C).

In both hemispheres, the motor cortex was more severely affected than the anterior frontal cortex (fig $3 \mathrm{D}-\mathrm{G}$ ). It was not possible to determine any difference in the amount of tau pathology between the right and the left frontal lobes due to differences in processing and no absolute neuronal counts were obtained.

\section{COMMENT}

We used fluid registered serial MRI to localise patterns of atrophy in vivo for the first time in PSP. The regions undergoing rapid atrophy closely reflected the anatomical location of cell loss and tau positive NFT load seen at post mortem.

A key feature of neurodegeneration is an increased rate of neuronal destruction. The increased rate of loss largely distinguishes neurodegeneration from normal ageing and the topography of loss defines the clinical features.

Previous imaging studies in PSP have been cross-sectional. The limitation of these studies is that they can at best imply rates of loss; similarly area or linear measures cannot fully capture volume changes as these are confounded by differences or changes in shape. Despite these points, such studies are helpful guides to the interpretation of MRI in a clinical context.

The strength of this case study is therefore in longitudinal imaging that showed increased rates of atrophy in life are closely associated with neurodegeneration and tau deposition in PSP. The distribution of cortical pathology in this case corresponds with previous studies ${ }^{12}{ }^{13}$ which suggested that atrophy is greatest in the posterior frontal lobes in PSP. In this case, the motor cortex was pathologically more severely affected than the anterior frontal cortex and this is reflected in the volume changes seen on serial imaging. This supports the theory that cortical atrophy observed on in vivo imaging in PSP is due to primary pathology rather than deafferentation secondary to loss of projecting neurones.

Despite a 19 month interval between the final MRI scan and death in this case, a close correlation was seen between volume loss in vivo and pathology at autopsy. This might suggest that either frontal lobe atrophy occurs early in the course of the disease as suggested in previous cross-sectional studies ${ }^{14}$ or that the pattern of volume loss remains consistent into the latter stages of the disease. Serial imaging in the early stages may show accelerated rates of atrophy in areas specific to PSP and this technique may prove useful in differentiating PSP from other akinetic-rigid syndromes. This is supported by the results of a recent case report using fluid 

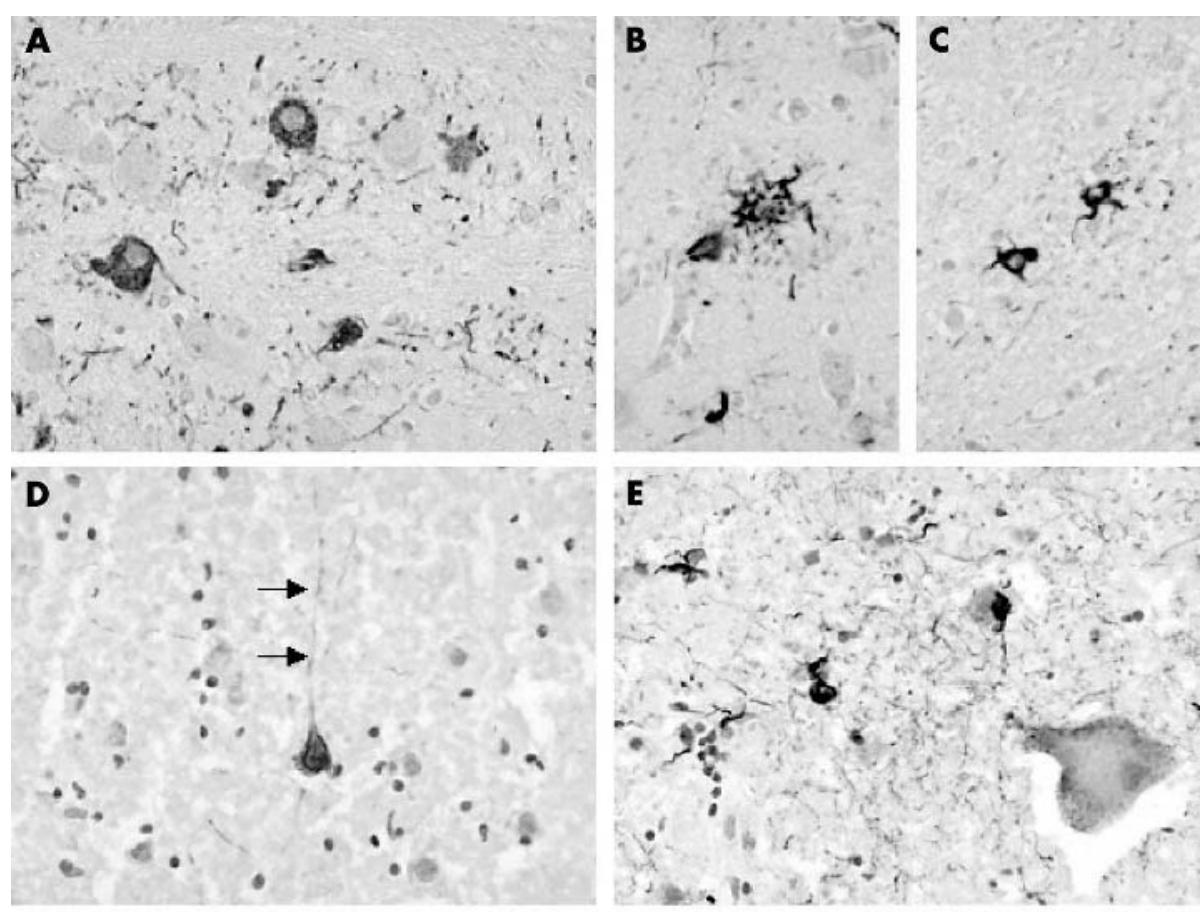

Figure 3 Pathological features of progressive supranuclear palsy are illustrated by immunohistochemical staining for tau. (A) Neurofibrillary tangles and neuropil threads are present in the pontine base. (B) Tufted astrocytes are demonstrated in the anterior frontal cortex and (C) oligodendroglial inclusions in the cerebellar white matter. Comparison of the anterior frontal $(D, F)$ and motor cortices $(E, G)$ in each hemisphere shows more numerous tau positive structures including glial inclusions and neuropil threads in the motor cortex than in the corresponding anterior frontal cortex (arrows on D pointing to the apical dendrite of a tau positive neurone). D, E show frozen sections from the right half brain, while $F, G$ illustrate paraffin embedded tissue from the left hemisphere (magnification

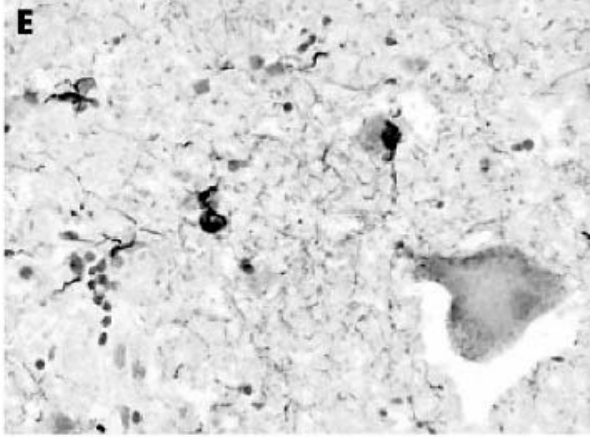
$\times 40$ ).
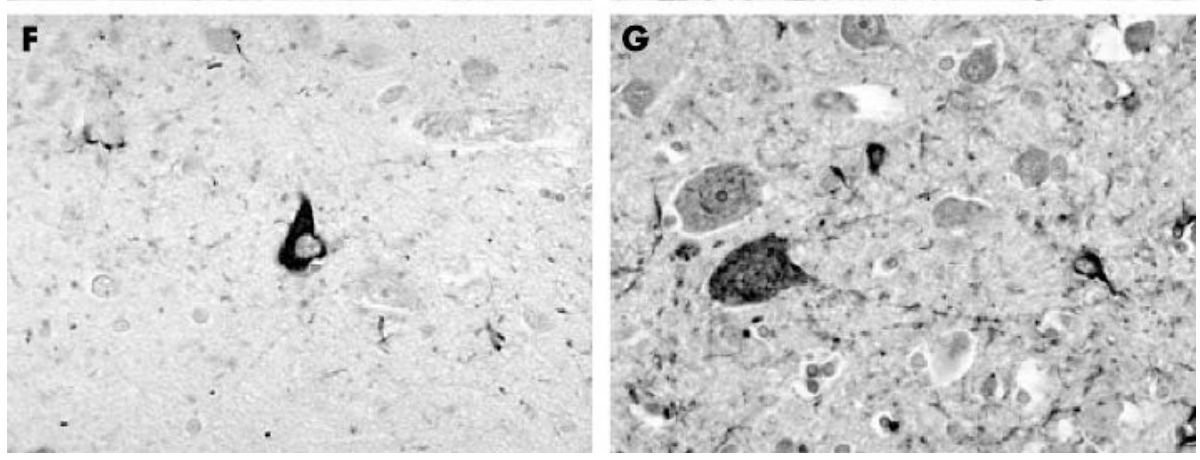

registration to demonstrate greatest rates of atrophy in the pons and cerebellum in a case of confirmed multiple system atrophy with parkinsonism and cognitive impairment. ${ }^{15}$

Registration based measures of atrophy rates track early disease progression ${ }^{16}$ and therefore may be a useful tool to assess the efficacy of potential disease modifying treatments. The observation in this case that regional atrophy in life is associated with pathological load lends support to the use of serial imaging as a surrogate marker of progression.

\section{Authors' affiliations}

D C Paviour, A J Lees, The Sara Koe PSP Research Centre, Institute of Neurology UCL, London UK

J M Schott, M N Rossor, N C Fox, Dementia Research Group (UCL), Institute of Neurology, London, UK

J M Stevens, Department of Clinical Neuroradiology, National Hospital for Neurology and Neurosurgery, London, UK

T Revesz, J L Holton, Department of Molecular Pathogenesis, Queen Square Brain Bank and Division of Neuropathology, Institute of Neurology, UCL, London, UK

M N Rossor, Division of Neuroscience and Psychological Medicine, Faculty of Medicine, Imperial College of Science, Engineering and Medicine, London, UK

DCP is supported by a grant from the PSP (Europe) Association; the imaging work is supported by a UK Medical Research Council (MRC) Program grant G9626876.

NCF holds an MRC Senior Clinical Fellowship.
$\mathrm{JH}$ is partly supported by the Reta Lila Weston Institute of Neurological Sciences.

JMS is in receipt of an Alzheimer's Society Research Fellowship.

Competing interests: This article was submitted before Professor $M N$ Rossor become Editor of the Journal of Neurology, Neurosurgery, and Psychiatry and he had no involvement in the peer review or decision making process.

Correspondence to: Dr D C Paviour, The Sara Koe PSP Research Centre, Institute of Neurology, 1 Wakefield Street, London, WCIN IPJ, UK; dpaviour@dementia.ion.ucl.ac.uk

Received 8 December 2003

In revised form 16 March 2004

Accepted 21 March 2004

\section{REFERENCES}

1 Steele JC, Richardson JC, Olszewski J. Progressive supranuclear palsy. Arch Neurol 1964;10:333-59.

2 Nath U, Ben Shlomo Y, Thomson RG, et al. The prevalence of progressive supranuclear palsy (Steele-Richardson-Olszewski syndrome) in the UK. Brain 2001; 124:1438-49.

3 Hauw JJ, Daniel SE, Dickson D, et al. Preliminary NINDS neuropathologic criteria for Steele-Richardson-Olszewski syndrome (progressive supranuclear palsy). Neurology 1994;44:2015-19.

4 Lees AJ. The Steele Richardson Olszewski syndrome. In: Marsden CD, Fahn S, eds. Movement Disorders 2. London: Butterworths, 1987:272-87.

5 Litvan I, Agid Y, Jankovic J, et al. Accuracy of clinical criteria for the diagnosis of progressive supranuclear palsy (Steele-Richardson-Olszewski syndrome). Neurology 1996;46:922-30 
6 Schulz JB, Skalej M, Wedekind D, et al. Magnetic resonance imaging-based volumetry differentiates idiopathic Parkinson's syndrome from multiple system atrophy and progressive supranuclear palsy. Ann Neurol 1999;45:65-74

7 Schrag A, Good CD, Miszkiel K, et al. Differentiation of atypical parkinsonian syndromes with routine MRI. Neurology 2000;54:697-702.

8 Warmuth-Metz M, Naumann M, Csoti l, et al. Measurement of the midbrain diameter on routine magnetic resonance imaging: a simple and accurate method of differentiating between Parkinson disease and progressive supranuclear palsy. Arch Neurol 2001;58:1076-9.

9 Fox NC, Freeborough PA, Rossor MN. Visualisation and quantification of rates of atrophy in Alzheimer's disease. Lancet 1996;348:94-7.

10 Freeborough PA, Fox NC. Modeling brain deformations in Alzheimer disease by fluid registration of serial 3D MR images. J Comput Assist Tomogr $1998 ; 22: 838-43$.
11 Freeborough PA, Fox NC, Kitney RI. Interactive algorithms for the segmentation and quantitation of 3-D MRI brain scans. Comput Methods Programs Biomed 1997; 53:15-25.

12 Daniel SE, de Bruin VM, Lees AJ. The clinical and pathological spectrum of Steele-Richardson-Olszewski syndrome (progressive supranuclear palsy): a reappraisal. Brain 1995;118(Pt 3):759-70.

13 Verny M, Duyckaerts C, Agid Y, et al. The significance of cortical pathology in progressive supranuclear palsy. Clinico-pathological data in 10 cases. Brain 1996;119(Pt 4):1123-36.

14 Cordato NJ, Pantelis C, Halliday GM, et al. Frontal atrophy correlates with behavioural changes in progressive supranuclear palsy. Brain 2002; 125:789-800

15 Schott JM, Simon JE, Fox NC, et al. Delineating the sites and progression of in vivo atrophy in multiple system atrophy using fluid-registered MRI. Mov Disord 2003;18:955-8.

16 Schott JM, Fox NC, Frost C, et al. Assessing the onset of structural change in familial Alzheimer's disease. Ann Neurol 2003;53:181-8. 